\title{
Dispositional Mindfulness and Neural Correlates of Affect Regulation
}

\author{
Holly Hazlett-Stevens ${ }^{1, *}$ \\ ${ }^{1}$ Department of Psychology, University of Nevada, Reno, Reno, Nevada \\ "Corresponding author: Holly Hazlett-Stevens, Ph.D., Department of Psychology, Mail Stop 298, University of Nevada, Reno, NV 89557. Tel: +1-7756828702, E-mail: \\ hhazlett@unr.edu \\ Received 2017 February 02; Accepted 2017 June 06.
}

\begin{abstract}
Mindfulness meditation and mindfulness-based interventions offer numerous clinical benefits related to improved affect regulation and emotional functioning. Psychological and emotional benefits of dispositional mindfulness, conceptualized as an individual difference trait found among the meditation-naïve, also have been demonstrated. This literature review article describes five specific research investigations examining potential neurocognitive emotion regulation mechanisms associated with dispositional mindfulness. Higher dispositional mindfulness may allow for enhanced top-down prefrontal control of limbic activity when coping with negative emotional stimuli, and individuals with greater trait mindfulness also may experience lower levels of neural activity in response to such stimuli. These patterns of improved affect regulation with increased dispositional mindfulness may reflect lower resting activity in key brain areas such as the amygdala.
\end{abstract}

Keywords: Mindfulness, Emotions, Cognition, Neurosciences

\section{Dispositional Mindfulness and Neural Correlates of Affect Regulation}

The past decade has generated a proliferation of mindfulness research across a wide variety of disciplines, ranging from medicine and health care to psychiatry, psychology, and education. Mindfulness meditation first entered the field of Western medicine in 1979 when Jon KabatZinn, a meditation practitioner and medical researcher trained in molecular biology, opened a stress reduction clinic at the University of Massachusetts (UMass) Medical School to teach mindfulness meditation practices to medical patients. Kabat-Zinn captured the Buddhist origins of mindfulness with the following definition: "the awareness that emerges through paying attention on purpose, in the present moment, and nonjudgmentally to the unfolding of experience moment to moment" (1) (p. 145). The clinical benefits of his eight-week curriculum, now known as Mindfulness-Based Stress Reduction (MBSR), have been well documented in subsequent research. Additional applications of MBSR and its adaptations, such as Mindfulness-based cognitive therapy(MBCT), targeted psychiatric disorders of anxiety and depression, stress-related physical conditions, employees in stressful work environments, as well as caregivers, physicians, and other health care professionals themselves.

As the clinical benefits of mindfulness meditation practice became known, neuroscience researchers began to investigate possible neurocognitive mechanisms underlying observed symptom relief and stress reduction. In one example, participants exhibited improved orienting of attention following MBSR (2), providing evidence of improved attention regulation. Military participants who received a similar intervention known as Mindfulnessbased mind fitness training (MMFT) and regularly practiced mindfulness training as instructed demonstrated increased working memory capacity on an Ospan cognitive performance task (3). Mindfulness interventions also might influence neural circuitry associated with emotion regulation. After receiving MBSR to treat social anxiety disorder, participants exhibited decreased negative emotion, reduced amygdala activation, and increased activity in parietal and other regions important for deployment of attention when they were presented with negative selfbeliefs and instructed to engage a breath-focused attention task (4). In contrast, distraction-focused attention task instructions did not produce the same emotion regulation effects. In a subsequent randomized controlled trial for social anxiety disorder (5), MBSR led to: 1) decreased negative emotion when faced with negative selfbeliefs during a receptive awareness attention regulation task, and 2) increased activation in attention-related parietal cortex regions when compared to an aerobic exercise stress reduction control group. Another way MBSR may improve emotion regulation is by enhancing attentional focus on present-moment direct sensory experience rather than elaborative self-referential narratives. Farb et al. (6) compared participants who recently completed MBSR to non-meditators using an experiential attention focus laboratory task. Functional magnetic resonance imaging (fMRI) data showed that MBSR participants exhibited 
notable reductions in medial prefrontal cortex (PFC) regions and increased lateral PFC and viscerosomatic areas (such as the insula) during the experiential focus task, and functional connectivity between the right insula and the medial PFC found in non-meditators was uncoupled for the MBSR group. Taken together, these preliminary studies suggest that mindfulness-based interventions may improve attention regulation, reduce negative emotional reactivity, and facilitate adaptive emotion regulation.

In a concurrent line of research, neuroscientists interested in the effects of various forms of meditation discovered greater thickness in certain cortical areas of the brain for experienced meditators compared to non-meditators. For example, Lazar et al. (7) employed magnetic resonance imaging (MRI) technology and found that brains of meditators averaging nine years of daily meditation practice were significantly thicker in right anterior insula and prefrontal regions than brains of non-meditators. Experienced Zen meditators had lower pain sensitivity as well as thicker cortex in the dorsal anterior cingulate and bilaterally in secondary somatosensory cortex than control participants; lower pain sensitivity was associated with cortical thickness in pain-related brain areas including the anterior insula, anterior cingulate cortex, and bilateral parahippocampal gyrus across both groups of participants (8). In another pain study, long-term meditators reported reduced unpleasantness, but not intensity, of painful stimuli compared to novices while practicing an "open monitoring" form of meditation; these results were not found during a "focused attention" meditation (9). Other research examining EEG activity (10) found marked gamma wave activity among experienced Tibetan Buddhist practitioners engaging compassion meditation in the laboratory. In sum, neuroscience research efforts such as these have established a biological basis for the subjective benefits meditators reportedly have enjoyed over thousands of years.

\section{Dispositional Mindfulness}

A final approach to mindfulness research has examined whether mindfulness can be measured as a naturally occurring personal trait outside the meditation context. Rather than investigating various effects of mindfulness training, researchers conceptualize mindfulness as a dispositional trait. In this area of research, the degree to which individuals report experiences of mindfulness over the course of daily life is conceptualized as an individual difference variable and measured with self-report questionnaires. The mindful attention awareness scale (MAAS) (11) is a widely-used example. Based on a definition of mindfulness as present-centered attention and awareness, the MAAS was developed to assess individual differences in the self-reported frequency of mindfulness states over time. This 15-item measure contains reverse-worded items (e.g., "I find it difficult to stay focused on what's happening in the present"), to which participants indicate the frequency of each on a 1 ("almost always") to 6 ("almost never") Likert scale. Thus, higher MAAS scores are considered to reflect higher levels of trait mindfulness. Previous research with various groups of participants has shown that MAAS scores yield good psychometric properties of convergent, discriminant, and criterion validity as well as consistent and reliable unidimensional factor structure, temporal stability, test-retest reliability and agreement, and internal consistency ( $\alpha$ coefficients ranging from .82 .87). In addition, MAAS scores correlated positively with various self-report measures indicating psychological well-being and correlated negatively with several measures of clinical symptoms and distress. Also as expected, Zen center practitioners scored higher on the MAAS than a matched comparison group, and increased mindfulness following a mindfulness-based intervention for cancer patients was related to reduced psychological disturbance. MAAS scores also predicted lower cortisol responses, as well as lower anxiety and negative affect, when participants encountered a laboratory Trier social stress task (TSST) (12).

Alternative self-report measures of dispositional mindfulness are based upon a multifaceted approach. A leading example, the five facet mindfulness questionnaire (FFMQ) (13), contains items from the MAAS and four other mindfulness questionnaires to capture additional aspects of mindful experience, including: 1) non-reactivity to inner experience, 2) observing inner experience, 3) acting with awareness (as measured primarily by the MAAS), 4) describing experience with words, and 5) non-judging of experience. Factor analysis of the FFMQ supported this fivefactor structure, although the facet of observing inner experience only fit the overall hierarchical model with meditating participants. FFMQ subscale scores correlated as expected with self-report measures of other psychological constructs, and increases across these mindfulness facets following intervention have been found consistently in randomized controlled trials of mindfulness-based interventions (14).

Neuroscience researchers recently have examined relationships between self-reported dispositional mindfulness and neural networks believed to underlie affect regulation. This is an important development, not only to provide validity for the conceptualization and self-report measurement of dispositional mindfulness, but also to establish whether such naturally occurring individual differences are associated with adaptive neurocognitive functioning. Therefore, the purpose of this review is to exam- 
ine available neuroscience research investigations of dispositional mindfulness and affect regulation. Evidence that certain non-meditating individuals naturally exhibit strengths and abilities previously associated with mindfulness training could provide further insight into the neural and cognitive mechanisms of mindfulness interventions and deepen our scientific understanding of mindfulness phenomena.

\section{Dispositional Mindfulness and Affect Labeling}

Creswell et al. (15) examined associations between dispositional mindfulness and neural mechanisms of emotional regulation during an affect labeling laboratory task. Based on their observation that mental labeling of direct emotional experience with words is a common mindfulness meditation technique, they developed an analogous affect labeling task in which participants viewed images of emotionally expressive faces and chose the appropriate affect label from a pair of affect words appearing at the bottom of the screen. Given previous research findings that affect labeling activated right ventrolateral and medial prefrontal cortex (PFC) areas while reducing amygdala responses (16), Creswell et al. proposed that dispositional mindfulness could be associated with greater PFC activation coupled with reduced amygdala activation when participants engaged the affect labeling task. This predicted pattern of brain activity during the affect labeling task would suggest improved emotion regulation theoretically due to more effective top-down PFC inhibition of limbic responses. A total of 27 healthy undergraduate students completed the MAAS and five other individual difference selfreport measures, followed by the affect labeling task and a gender labeling control task administered during fMRI data acquisition.

Results indicated a weak and statistically nonsignificant relationship in the predicted direction between dispositional mindfulness and affect labeling task reaction times $(\mathrm{r}=-0.29 ; \mathrm{P}=0.16)$ and no association between dispositional mindfulness and the gender labeling control task reaction times $(r=0.002)$; error rates were not examined due to the small number of errors produced by both tasks. Although dispositional mindfulness was unrelated to ethnicity and to all other self-report variables except one (public self-consciousness; $r=-0.51 ; \mathrm{P}=0.01$ ), the authors used self-report measures and gender as control variables in subsequent analyses. Contrasts comparing neural activity during the affective labeling task to the gender labeling task revealed that higher dispositional mindfulness was positively associated with activity throughout the PFC during affect labeling compared with gender labeling. More specifically, greater dispositional mindfulness was associated with greater activity in the following areas: right ventrolateral PFC, left ventrolateral PFC, ventromedial PFC, medial PFC, right dorsolateral PFC, and the left insula. In addition, dispositional mindfulness was associated with lower bilateral amygdala activity during affect labeling compared with gender labeling. Further analyses examining mindfulness and the connectivity between PFC regions and the amygdala compared participants high versus low in dispositional mindfulness using a median split of MAAS scores. Strong negative associations between several PFC regions and the amygdala were found only among high mindfulness participants (e.g., right ventrolateral PFC and right amygdala, ventromedial PFC and right amygdala, medial PFC and right amygdala, right dorsolateral PFC and right amygdala). None of these associations were found in the low mindfulness group, and these correlational effects for the high mindfulness group were significantly different from those obtained for the low mindfulness group. Creswell et al. interpreted the observed associations between dispositional mindfulness and PFC activation, coupled with amygdala deactivation, during affect labeling as evidence that individuals reporting greater mindfulness exhibited improved top-down PFC inhibition of limbic responses. The pronounced negative relationships between PFC and amygdala regions found only among the high mindfulness participants provided further support that such neural emotion regulation pathways are enhanced for individuals high in trait mindfulness. Thus, mindfulness may reduce the intensity and duration of automatic negative affectivity via enhanced top-down emotion regulation neural networks.

\section{Dispositional Mindfulness and Cognitive Reap- praisal}

Dispositional mindfulness also may enhance topdown cortical regulation of negative affect during the deliberate cognitive reappraisal of emotional stimuli. Drawing from previous research demonstrating that reappraisal of negative emotion elicited increased prefrontal and decreased amygdala activity (17), Modinos et al. (18) administered a dispositional mindfulness self-report measure to 18 healthy university students before they engaged in fMRI cognitive reappraisal and attention tasks. Dispositional mindfulness was measured with the total score from Kentucky inventory of mindfulness skills (KIMS) (19), a 39-item questionnaire with demonstrated validity and good reliability that preceded development of the FFMQ. The KIMS is comprised of separate subscales covering four aspects of mindfulness, including 1) observing direct experience, 2) describing private experience, 3) acting 
with awareness, and 4) accepting experience without judgment. In this experiment, reappraisal was defined as the cognitive reinterpretation of negative stimuli to decrease aversive emotional experience. After completing the KIMS and before scanning, participants were trained in various reappraisal strategies in the laboratory. These strategies included: 1 ) creating a positive outcome to a negative situation, 2) transforming the scene into a positive circumstance, and 3) objectifying the scene depicted in the picture by recognizing it was only a picture representation. During the fMRI session, participants then viewed negative and neutral valence pictures from the International affective picture system (IAPS) (20). During these trials, participants received instructions either to reinterpret the content of a negative picture so that it no longer elicited a negative response (Reappraise) or to allow themselves to experience any natural emotional responses to neutral and negative valence pictures (Attend). Experimental task trials therefore were comprised of three main conditions: neutral (Attend), negative (Attend), and Reappraise.

As expected, results indicated higher negative emotion ratings after the negative attend trials, and these ratings diminished following reappraisal trials. A variable of reappraisal success was created by subtracting Reappraise negative emotion ratings from Negative Attend negative emotion ratings. Reappraisal success was positively correlated with dispositional mindfulness $(\mathrm{r}=0.65, \mathrm{P}=0.005$, twotailed), and no gender differences were found in either variable. Imaging data analyses revealed that when activity associated with negative attend trials was subtracted from activity associated with reappraisal trials, significant activation in left dorsolateral and right dorsomedial PFC, bilateral inferior frontal gyrus, and dorsal anterior cingulate cortex (ACC) was associated with reappraisal. Activity in both the left and right dorsolateral PFC regions positively correlated with reappraisal success (left, $\mathrm{r}=0.50, \mathrm{P}$ $=0.03$; right, $\mathrm{r}=0.47, \mathrm{P}=0.05$; two-tailed). Significant activation of the left amygdala was found during Negative Attend trials, and this activation was inversely correlated with right dorsomedial PFC activation during reappraisal. Total dispositional mindfulness scores were positively correlated with right dorsomedial PFC activation during reappraisal, in addition to the other prefrontal areas of interest activated in reappraisal. Thus, mindfulness was associated with increased right dorsomedial PFC activation during reappraisal, which in turn was associated with decreased amygdala activation during Negative Attend trials, but dispositional mindfulness was not directly correlated with amygdala responses to Negative Attend trials. Modinos et al. therefore concluded that the recruitment of PFC areas to reduce amygdala reactivity found during reappraisal trials was enhanced by increased dispositional mindfulness. The dorsolateral and dorsomedial PFC regions activated in the reappriasal task of this experiment are considered important to the down-regulation of negative emotion because these areas previously have been associated with higher-order self-referential processing (21), the evaluation of self-referential stimuli (22), and judgments of emotional stimuli (23). Furthermore, the dorsal ACC region activated during reappraisal appears to serve a cognitive control function in the regulation of emotion (24). Higher dispositional mindfulness, at least when measured with a multidimensional scale such as the KIMS, may reflect increased cognitive control via successful reappraisal as supported by strengthened underlying emotional regulation neural circuitry.

\section{Dispositional Mindfulness and Evaluation of Affec- tive Stimuli}

A subsequent investigation demonstrated that dispositional mindfulness was associated with the early processing of emotional stimuli as well. Brown et al. (25) employed EEG recording procedures to examine the late positive potential (LPP) of the event-related potential to visual stimuli that varied in emotional valence and arousal. Based on previous research that found the LPP range to be sensitive to the evaluation and downregulation of such stimuli (26), Brown et al. examined associations between dispositional mindfulness and this index of initial attentional engagement of emotional images. They reasoned that if established associations between mindfulness training and improved emotion regulation also hold for meditation-naïve dispositionally mindful individuals, this LPP neural marker of emotional arousal in response to emotionally evocative pictures should be lower as the level of dispositional mindfulness increases. A total of 34 healthy introductory psychology students completed both the MAAS and the FFMQ dispositional mindfulness measures before study procedures. Additional self-report measures of attentional control, neuroticism, and negative affectivity were included in the questionnaire battery. During EEG recording, all participants passively viewed 150 IAPS images consisting of: 1) 30 pleasant, high arousal images, 2) 30 pleasant, low arousal images, 3) 30 unpleasant, high arousal images, 4) 30 unpleasant, low arousal images, and 5) 30 neutral images. Task instructions were simply to look at each picture for its entire presentation duration.

As expected, both unpleasant and pleasant high arousal images elicited larger LPP responses than low arousal images. High arousal unpleasant images elected larger LPP responses than high arousal pleasant images, 
and low arousal unpleasant images did not yield significantly different responses than low arousal pleasant images did. Mindfulness measures included MAAS scores and the act with awareness FFMQ subscale scores, and these variables were strongly correlated with each other ( $\mathrm{r}=0.92 ; \mathrm{P}<0.0001)$ and with the attentional control measure (MAAS: $\mathrm{r}=0.60, \mathrm{P}<0.0002$; FFMQ: $\mathrm{r}=0.68$, $\mathrm{P}<0.0001)$. Repeated measures mixed model analysis revealed a significant main effect for MAAS scores on LPP amplitude qualified by a significant mindfulness by stimulus condition interaction, such that increased dispositional mindfulness of participants was associated with lower LPP responses to high arousal unpleasant images. Similar findings resulted with the FFMQ act with awareness subscale measure, and both MAAS and FFMQ measures strongly inversely correlated with the LPP amplitude elicited by unpleasant images. This same pattern of results was found when the analysis was repeated while controlling for attentional control, and attentional control did not predict LPP responses. Dispositional mindfulness also was associated with lower LPP responses to pleasant high arousal erotica images. Self-report measures of the psychological traits inversely correlated with mindfulness - neuroticism and negative affectivity - were associated with higher LPP responses to high arousal unpleasant images. This focus on the LPP of ERP methodology allowed researchers to investigate how dispositional mindfulness modulates neural responses in the early phase of processing emotional stimuli. The lower LPP responses to arousing unpleasant images among more mindful participants suggested that previously observed emotional advantages of dispositional mindfulness take place early in the emotion regulation process, soon after stimulus contact. This individual difference effect also was observed in response to arousing positive stimuli and does not appear to be merely a function of attentional control.

\section{Non-reactivity Mindfulness Facet and Negative Bias}

Mindful individuals also might regulate emotional responses to negative stimuli via interoception marked by reduced insula activation. In an investigation of dispositional mindfulness and the cognitive bias for negative content associated with depression, Paul et al. (27) presented a go/no-go emotional inhibition task to 19 healthy male participants. After completing the FFMQ and measures of depression, rumination, and perceived stress, participants completed separate fMRI experimental sessions on two different days. During each session, participants completed either a mental arithmetic stress-induction task or a mindful breathing task immediately before performing the go/no-go task. During the go/no-go task, participants viewed emotionally neutral face images, emotionally negative face images, and scrambled images, and they were instructed to press a button for scrambled images and one type of image while inhibiting their response to the remaining type of image.

As predicted, participants exhibited poorer inhibitory responses to negative than to neutral stimuli. Of the five FFMQ subscales, only non-reactivity subscale scores correlated inversely with rumination and perceived stress. Individuals higher in this aspect of dispositional mindfulness showed slower respiration rate during stress induction, and non-reactivity scores inversely correlated with negative bias during the emotional inhibition task following stress induction but not following mindful breathing. Higher non-reactivity also correlated with better accuracy in response to negative images but not to neutral images. Neuroimaging data analysis revealed that higher non-reactivity was not associated with inferior frontal cortex area activation during the emotional inhibition task following mindful breathing, but non-reactivity was negatively correlated with activation in insula regions in response to negative trials. No correlations between brain activation during the emotional inhibition task and nonreactivity were found following stress induction. The authors concluded that the non-reactivity facet of dispositional mindfulness may protect against depression vulnerability due to a reduced negative cognitive bias and improved ability to inhibit responses to negative emotional information. This non-reactivity did not appear to involve frontal cortex activation, but rather was associated with reduced insula activity when encountering negative stimuli after the mindful breathing task. This pattern of results suggests that the non-reactivity aspect of mindfulness may reduce initial emotional responding to negative stimuli rather than reflect a top-down effortful regulation process.

\section{Dispositional Mindfulness, Reactivity, and Resting Amygdala Activity}

This reduced emotional and neural reactivity associated with dispositional mindfulness also may reflect underlying resting neural activity. Way et al. (28) collected MAAS and self-report measures of depression, trait anxiety, neuroticism, and public self-consciousness from 27 healthy undergraduate students before undergoing fMRI laboratory tasks. During the experiment, participants passively viewed a fixation cross, engaged in a neutral shapematching task, and engaged in a face-matching task containing pictures of faces displaying emotional expressions. Given previous research demonstrating elevated amygdala resting activity in depression, Way et al. reasoned that 
dispositional mindfulness may protect individuals from depression not only via reduced amygdala reactivity to emotional stimuli, but also with lower levels of resting amygdala activity.

Resting state neuroimaging analyses involving the fixation cross revealed that depression scores positively correlated with right amygdala activation among other areas. In contrast, dispositional mindfulness negatively correlated with activity in several brain areas, including bilateral amygdala and hippocampal areas. Furthermore, reactivity analyses involving the emotional face-matching task showed that dispositional mindfulness negatively correlated with amygdala activation, whereas depression positively correlated with amygdala activation. When comparing amygdala responses to the emotional faces to amygdala activity while passively viewing the fixation cross, no significant relationship between dispositional mindfulness and amygdala activation resulted. However, the positive correlation between depression and amygdala activation in response to emotional faces persisted when compared to the fixation cross. The authors concluded that depression may be characterized both by increased amygdala activity in response to emotional stimuli as well as during resting activity. Dispositional mindfulness may protect individuals from depression via reduced amygdala reactivity to emotional stimuli, but this individual difference seems largely driven by underlying differences in the resting state of the amygdala. Furthermore, depression positively correlated with medial prefrontal regions during rest associated with self-referential processing, or the cognitive processing of self-relevant information. Conversely, higher dispositional mindfulness was associated with lower levels of resting activation in medial prefrontal and parietal self-referential areas. This final investigation raises the intriguing possibility that dispositional mindfulness facilitates adaptive emotion regulation not only by modulating responses to emotional events, but also by maintaining lower levels of associated neural activity during rest.

\section{Summary and Conclusions}

A growing body of psychological research links dispositional mindfulness to increased emotional well-being and happiness coupled with lower levels of perceived stress, depression, and anxiety. The recent research investigations reviewed here explored the potential neural mechanisms responsible for these observed emotional benefits. Individuals high in dispositional mindfulness may enjoy improved neural affect regulation while verbally labeling facial expressions of emotion (15) and while actively reappraising negative valence images (18). When confronted with such stimuli, mindful participants exhibited enhanced prefrontal cortical downregulation of negative affective responding and reduced amygdala activity. This reduced neural activity in response to arousing emotional imagery among mindful individuals appears to occur in the early phases of active processing (25) and may reflect associations between the "non-reactivity" facet of dispositional mindfulness and reduced insula activation while inhibiting responses to negative stimuli, at least after mindful breathing (27). Both potential emotion regulation mechanisms - top-down prefrontal cortical control of negative emotion and associated limbic regions (e.g., amygdala) as well as reduced emotional and neural reactivity upon encountering emotional stimuli may be supported by an underlying tendency toward lower resting amygdala activity among dispositionally mindful individuals (28). Indeed, dispositional mindfulness appears associated with other resting-state neural individual differences, including: 1) inter-network intrinsic functional connectivity believed to underlie an ability to attend to present-moment direct experience without judgment (29), 2) increased regional homogeneity across key emotional processing brain regions reflecting improved synchronization of spontaneous brain activity (30), and 3) decreased grey matter volume in the right amygdala (31). Despite valid criticisms that self-report questionnaires including the MAAS may not reflect true original meanings of the word "mindfulness" (32), this individual difference variable appears related to neural circuitry crucial for effective emotion regulation.

\section{References}

1. Kabat-Zinn J. Mindfulness-Based Interventions in Context: Past, Present, and Future. Clin Psychol Sci Pract. 2006;10(2):144-56. doi 10.1093/clipsy.bpg016.

2. Jha AP, Krompinger J, Baime MJ. Mindfulness training modifies subsystems of attention. Cogn Affect Behav Neurosci. 2007;7(2):109-19. [PubMed: 17672382].

3. Jha AP, Stanley EA, Kiyonaga A, Wong L, Gelfand L. Examining the protective effects of mindfulness training on working memory capacity and affective experience. Emotion. 2010;10(1):54-64. doi: 10.1037/a0018438. [PubMed: 20141302].

4. Goldin PR, Gross JJ. Effects of mindfulness-based stress reduction (MBSR) on emotion regulation in social anxiety disorder. Emotion. 2010;10(1):83-91. doi: 10.1037/a0018441. [PubMed: 20141305].

5. Goldin P, Ziv M, Jazaieri H, Hahn K, Gross JJ. MBSR vs aerobic exercise in social anxiety: fMRI of emotion regulation of negative self-beliefs. Soc Cogn Affect Neurosci. 2013;8(1):65-72. doi: 10.1093/scan/nss054. [PubMed: 22586252].

6. Farb NA, Segal ZV, Mayberg H, Bean J, McKeon D, Fatima Z, et al. Attending to the present: mindfulness meditation reveals distinct neural modes of self-reference. Soc Cogn Affect Neurosci. 2007;2(4):313-22. doi: 10.1093/scan/nsm030. [PubMed: 18985137].

7. Lazar SW, Kerr CE, Wasserman RH, Gray JR, Greve DN, Treadway MT, et al. Meditation experience is associated with increased cortical thickness. Neuroreport. 2005;16(17):1893-7. [PubMed: 16272874]. 
8. Grant JA, Courtemanche J, Duerden EG, Duncan GH, Rainville P. Cortical thickness and pain sensitivity in zen meditators. Emotion. 2010;10(1):43-53. doi: 10.1037/a0018334. [PubMed: 20141301].

9. Perlman DM, Salomons TV, Davidson RJ, Lutz A. Differential effects on pain intensity and unpleasantness of two meditation practices. Emotion. 2010;10(1):65-71. doi: 10.1037/a0018440. [PubMed: 20141303].

10. Lutz A, Greischar LL, Rawlings NB, Ricard M, Davidson RJ. Long-term meditators self-induce high-amplitude gamma synchrony during mental practice. Proc Natl Acad Sci U S A. 2004;101(46):16369-73. doi: 10.1073/pnas.0407401101. [PubMed: 15534199].

11. Brown KW, Ryan RM. The benefits of being present: mindfulness and its role in psychological well-being.JPers Soc Psychol. 2003;84(4):82248. [PubMed: 12703651].

12. Brown KW, Weinstein N, Creswell JD. Trait mindfulness modulates neuroendocrine and affective responses to social evaluative threat. Psychoneuroendocrinology. 2012;37(12):2037-41. doi: 10.1016/j.psyneuen.2012.04.003. [PubMed: 22626868].

13. Baer RA, Smith GT, Hopkins J, Krietemeyer J, Toney L. Using self-report assessment methods to explore facets of mindfulness. Assessment. 2006;13(1):27-45. doi: 10.1177/1073191105283504. [PubMed: 16443717].

14. Quaglia JT, Braun SE, Freeman SP, McDaniel MA, Brown KW. Metaanalytic evidence for effects of mindfulness training on dimensions of self-reported dispositional mindfulness. Psychol Assess. 2016;28(7):803-18. doi: 10.1037/pas0000268. [PubMed: 27078183].

15. Creswell JD, Way BM, Eisenberger NI, Lieberman MD. Neural correlates of dispositional mindfulness during affect labeling. Psychosom Med. 2007;69(6):560-5. doi: 10.1097/PSY.0b013e3180f6171f. [PubMed: 17634566].

16. Lieberman MD, Eisenberger NI, Crockett MJ, Tom SM, Pfeifer JH, Way BM. Putting feelings into words: affect labeling disrupts amygdala activity in response to affective stimuli. Psychol Sci. 2007;18(5):421-8. doi: 10.1111/j.1467-9280.2007.01916.x. [PubMed: 17576282].

17. Ochsner KN, Gross JJ. The cognitive control of emotion. Trends Cogn Sci. 2005;9(5):242-9. doi: 10.1016/j.tics.2005.03.010. [PubMed: 15866151].

18. Modinos G, Ormel J, Aleman A. Individual differences in dispositional mindfulness and brain activity involved in reappraisal of emotion. Soc Cogn Affect Neurosci. 2010;5(4):369-77. doi: 10.1093/scan/nsq006. [PubMed: 20147457].

19. Baer RA, Smith GT, Allen KB. Assessment of Mindfulness by SelfReport: The Kentucky Inventory of Mindfulness Skills. Assessment. 2004;11(3):191-206.

20. Lang PJ, Bradley MM, Cuthbert BN. International affective picture system (IAPS): Technical manual and affective ratings. Gainesville: The Center for Research in Psychophysiology, University of Florida; 1999.

21. Northoff G, Heinzel A, de Greck M, Bermpohl F, Dobrowolny H, Panksepp J. Self-referential processing in our brain-a meta-analysis of imaging studies on the self. Neuroimage. 2006;31(1):440-57. doi: 10.1016/j.neuroimage.2005.12.002. [PubMed:16466680].

22. Gusnard DA, Akbudak E, Shulman GL, Raichle ME. Medial prefrontal cortex and self-referential mental activity: relation to a default mode of brain function. Proc Natl Acad Sci U S A. 2001;98(7):4259-64. doi: 10.1073/pnas.071043098. [PubMed: 11259662].

23. Northoff G, Heinzel A, Bermpohl F, Niese R, Pfennig A, Pascual-Leone A, et al. Reciprocal modulation and attenuation in the prefrontal cortex: an fMRI study on emotional-cognitive interaction. Hum Brain Mapp. 2004;21(3):202-12. doi: 10.1002/hbm.20002. [PubMed: 14755839].

24. Lane RD, Reiman EM, Axelrod B, Yun LS, Holmes A, Schwartz GE. Neural correlates of levels of emotional awareness. Evidence of an interaction between emotion and attention in the anterior cingulate cortex. J Cogn Neurosci. 1998;10(4):525-35. [PubMed: 9712681].

25. Brown KW, Goodman RJ, Inzlicht M. Dispositional mindfulness and the attenuation of neural responses to emotional stimuli. Soc Cogn Affect Neurosci. 2013;8(1):93-9. doi: 10.1093/scan/nss004. [PubMed: 22253259].

26. Foti D, Hajcak G. Deconstructing reappraisal: descriptions preceding arousing pictures modulate the subsequent neural response. J Cogn Neurosci. 2008;20(6):977-88. doi: 10.1162/jocn.2008.20066. [PubMed: 18211235].

27. Paul NA, Stanton SI, Greeson JM, Smoski MJ, Wang L. Psychological and neural mechanisms of trait mindfulness in reducing depression vulnerability. Soc Cogn Affect Neurosci. 2013;8(1):56-64. doi: 10.1093/scan/nss070. [PubMed: 22717383].

28. Way BM, Creswell JD, Eisenberger NI, Lieberman MD. Dispositional mindfulness and depressive symptomatology: correlations with limbic and self-referential neural activity during rest. Emotion. 2010;10(1):12-24. doi: 10.1037/a0018312. [PubMed: 20141298].

29. Doll A, Holzel BK, Boucard CC, Wohlschlager AM, Sorg C. Mindfulness is associated with intrinsic functional connectivity between default mode and salience networks. Front Hum Neurosci. 2015;9:461. doi: 10.3389/fnhum.2015.00461. [PubMed: 26379526].

30. Kong F, Wang X, Song Y, Liu J. Brain regions involved in dispositional mindfulness during resting state and their relation with well-being. Soc Neurosci. 2016;11(4):331-43. doi: 10.1080/17470919.2015.1092469. [PubMed: 26360907].

31. Taren AA, Creswell JD, Gianaros PJ. Dispositional mindfulness covaries with smaller amygdala and caudate volumes in community adults. PLoS One. 2013;8(5):e64574. doi:10.1371/journal.pone.0064574. [PubMed: 23717632].

32. Grossman P. Defining mindfulness by how poorly I think I pay attention during everyday awareness and other intractable problems for psychology's (re)invention of mindfulness: comment on Brown et al. (2011). Psychol Assess. 2011;23(4):1034-40. doi: 10.1037/a0022713. [PubMed: 22122674] discussion 1041-6. 